\title{
Simultaneous Travel and Hoist Maneuver Input Shaping Control Using Frequency Modulation
}

\author{
Sameer Arabasi ${ }^{1}$ and Ziyad Masoud ${ }^{2}$ \\ ${ }^{1}$ Department of Physics, German-Jordanian University, Amman 11180, Jordan \\ ${ }^{2}$ Department of Mechanical Engineering, German-Jordanian University, Amman 11180, Jordan \\ Correspondence should be addressed to Ziyad Masoud; zmasoud@vt.edu
}

Received 21 March 2017; Accepted 2 May 2017; Published 12 June 2017

Academic Editor: Francesco Pellicano

Copyright (c) 2017 Sameer Arabasi and Ziyad Masoud. This is an open access article distributed under the Creative Commons Attribution License, which permits unrestricted use, distribution, and reproduction in any medium, provided the original work is properly cited.

\begin{abstract}
This paper presents an input shaping control system for overhead crane operations involving simultaneous hoist and travel maneuvers. The control system utilizes model-based partial feedback linearization with frequency modulation. Traditional input shaping controllers target specific system frequencies. Therefore, they are incapable of accommodating the time dependant frequency associated with simultaneous hoist and travel crane maneuvers. Frequency modulation is used to tune the timedependent system frequency to the design frequency of a primary input shaping controller. Partial feedback linearization is used to eliminate the time-dependent damping of the system. The primary input shaper frequency is based on lowest operating frequency of the system associated with the longest hoisting cable length operation. Simulations results, using primary zero-vibration (ZV) and zero-vibration-derivative (ZVD) input shapers, are presented. General arbitrary input travel and hoist commands are simulated. Results demonstrate the ability of the proposed control system to eliminate residual oscillations in all simulated cases.
\end{abstract}

\section{Introduction}

Dynamics and control of cranes have been extensively researched in the past few decades $[1,2]$. This research was mainly targeting the reduction or elimination of residual vibrations and the reduction of maneuver time. Feedback control [3-5] is usually chosen over open-loop control systems for its robustness to modeling uncertainties. However, they need adding extra hardware components, some of which are expensive and challenging to implement, for example, motion sensors, oscillations angle sensors, and payload sway sensing systems. Such additions are not required for openloop control systems making it more attractive and cost effective.

One approach that uses open-loop control systems is command shaping. This approach is used for moving suspended objects and/or flexible systems [2]. Starr [6] developed a strategy for swing-free transport of suspended objects. Strip [7] showed that, for simply suspended objects, there is a family of acceleration strategies that are referred to as the double-step command shaping that results in a swing-free motion at a chosen velocity.

Input shaping is one of the most widely used command shaping techniques. Input shaping reduces the residual vibrations by convolving a sequence of carefully timed impulses with a general reference command signal. However it comes at a price of a large rise-time penalty $[8,9]$. Time delay filters where successfully implemented [10] to reduce jerks in input shapers for undamped and damped systems. Singhose et al. [11] compared smooth and nonsmooth commands by interpreting smooth commands as input-shaped functions. They concluded that S-curves smooth function must be four times slower than step commands shaped with zerovibration shapers to eliminate vibrations in single-mode systems. Erkorkmaz and Altintas [12] presented continuous position, velocity, and acceleration profiles by imposing limits on the first and second time derivatives of the feed rate. 
Alhazza and Masoud [13, 14] introduced a continuous wave-form command profile which eliminates residual vibrations in single-mode systems. They showed the effect of discretization of continuous commands on the system performance. The timing sensitivity of command shapers was affected by timing precision which is compromised by the sampling rates and the precision of switching times of the control hardware used. By incorporating a simulated feedback system in their command shaping algorithms, they were able to mitigate the effect of timing and their work was later extended to include the effect of damping $[15,16]$.

Including simultaneous travel and hoist maneuvers in the system model makes it mathematically more complex. Some researchers assume constant cable length to simplify the model. Such strategies depend on raising the payload, moving it horizontally, and lowering it to the final position. This results in a large time penalty. Implementing constant length input shapers in maneuvers that involve hoisting can lower the residual vibrations but cannot eliminate them [17]. However, research has been published that includes simultaneous travel and hoist in command shaping. Graphical profile generation [18], wave-form profiles [19], iterative learning control [20], and discrete-time command profiles [21] were all used to produce shaped commands for crane maneuvers involving simultaneous travel and hoist. More recently, Zhou et al. [22] used the concepts of equivalent frequency and the equivalent damping ratio to take into account the variable hoist length, and the optimal path planning is considered to avoid collision and improve efficiency. Abdullahi et al. [23] proposed an output-based command shaping (OCS) technique for an effective payload sway control of a crane with hoisting. The proposed technique is based on output signals of an actual system and reference model which can be utilized to minimize the hoisting effects on the payload sway.

The general theory of partial feedback linearization (PFL) for underactuated mechanical systems, such as the overhead cranes considered in this paper, was first developed by Spong [24]. Ever since, this technique has been the essence of many controllers developed and published in the literature. Cheng and Chen [25] used a controller, which combines a feedback linearization approach and a time delay control scheme. The time delay control completes the feedback linearization for a nonlinear system under the influence of uncertainty. Fang and Kelkar [26] used this technique for the fully nonlinear spacecraft model. Zhang et al. [27] proposed a nonlinear controller design scheme for a gantry crane system based on PFL. Park et al. [28] considered a PFL controller in a closedloop system that provides a simultaneous trolley position, sway suppression, and load hoisting control. Cho and Lee [29] proposed a controller using PFL with corrective control to compensate for system errors. Le et al. [30] proposed a nonlinear control scheme for an overhead crane based on the combination of two control design techniques, where PFL is mainly used for cargo swing control. The PFL approach is simple and easy to design and implement. However, it is not useful for systems with uncertainties.

In this work, a frequency modulation input shaping control scheme is proposed to allow for simultaneous travel and hoisting maneuvers in overhead cranes. The overhead

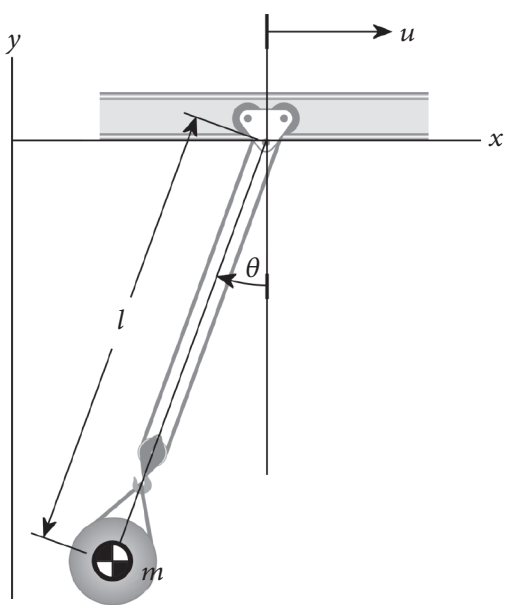

FIGURE 1: Overhead crane model.

crane maneuvers involving simultaneous hoist and travel represent a time varying system. This makes it a challenging problem in input shaping design since most input shaping techniques target specific discrete system frequencies. The proposed scheme is designed to accommodate continuously time varying system frequency introduced by the continuously varying hoisting cable length. The scheme allows for arbitrary joystick operator hoist and travel commands. This is demonstrated by running different simulations on a nonlinear model of the crane involving a series of random transfer maneuvers.

\section{Mathematical Model}

The overhead crane is modeled as a simple pendulum with a variable length hoisting cable. The payload of the crane is modeled as a rigid body of mass $m$ attached to the end of a massless inextensible rigid cable of variable length $l$, Figure 1 . The nonlinear equation of motion of the model is derived using the Lagrangian approach.

$$
\ddot{\theta}+2 \frac{\dot{l}}{\dot{l}} \dot{\theta}+\frac{g}{l} \sin \theta=\frac{\ddot{u}}{l} \cos \theta,
$$

where $u$ is the motion of the crane jib. For small oscillation angle $\theta,(1)$ is linearized as

$$
\ddot{\theta}+2 \frac{\dot{l}}{\dot{l}} \dot{\theta}+\frac{g}{l} \theta=\frac{\ddot{u}}{l} .
$$

Equation (2) represents a damped oscillator with a time varying frequency. The damping is a result of the time rate of change of the hoisting cable length. As a result, the system could exhibit negative damping during hoisting up maneuvers, which would increase the amplitude of the payload oscillations.

\section{Frequency Modulation Input Shaping}

Input shaping is a technique used to modify a general command signal by convolving the input signal with a sequence of 


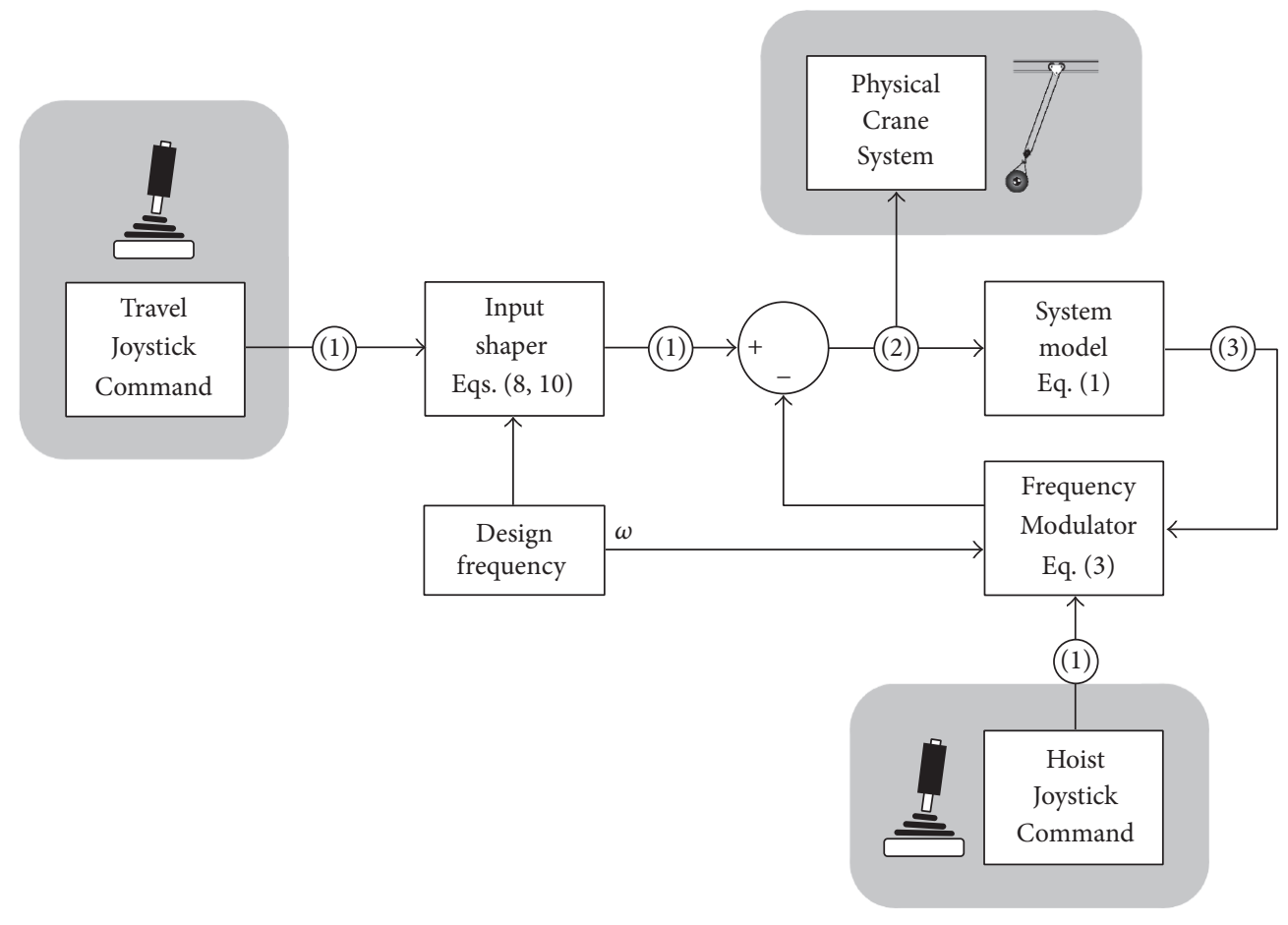
(1) Figure 5(a), Figure 6(a)
(3) Figure 5(d), Figure 6(d)
(2) Figure 5(c), Figure 6(c)

FIGURE 2: Schematic block diagram of the frequency modulation input shaping.

precisely timed impulses. The shaped command mitigates its own excited vibrations. Over the past three decades, numerous input shapers have been developed with different levels of robustness. Basic input shapers are designed for systems with specific constant frequency. Higher order shapers can accommodate sets of discrete frequencies; however, those shapers tend to significantly slow down the system [31].

3.1. Frequency Modulation. The large change in the length of the hoisting cable introduces a range of continuously varying frequencies. In this work, model-based frequency modulation (FM) is used to eliminate damping in the crane model and to limit its response to a constant design frequency, regardless of the hoisting cable length. This makes it possible to use a single frequency fast input shaping technique. The command signal to the plant of the feedback crane model is then used to drive the actual crane system, Figure 2.

The feedback control law used is

$$
\ddot{u}=-a \ddot{\theta}-b \dot{\theta},
$$

where $a$ and $b$ are time varying gains. Applying this control law to the linear equation of motion of the system, (2) yields

$$
\ddot{\theta}+\frac{2 \dot{l}+b}{l+a} \dot{\theta}+\frac{g}{l+a} \theta=0 .
$$

The gains $a$ and $b$ are chosen to reduce (4) to a simple harmonic oscillator model as

$$
\ddot{\theta}+\omega^{2} \theta=0 \text {. }
$$

The response frequency of the reduced model $\omega$ will be used to design a single frequency input shaper. The feedback gains $a$ and $b$ are determined by setting the coefficients of (4) to the coefficients of (5). The resulting formulae for the gains are

$$
\begin{aligned}
& a=\frac{g}{\omega^{2}}-l, \\
& b=-2 \dot{l} .
\end{aligned}
$$

3.2. Input Shaping. Ideally, any input shaping technique can be used to design a primary input shaper for the FM input shaping scheme. In this work, the FM input shaping control will be demonstrated using the zero-vibration (ZV) input shaping technique which is considered the fastest technique, and the more robust zero-vibration-derivative (ZVD) input shaping technique.

The $\mathrm{ZV}$ input shaping process is the convolution of two impulses with a general input command, Figure 3. The response to the second impulse of the $\mathrm{ZV}$ shaper mitigates the vibrations excited by the first impulse. The amplitudes of the impulse and their time locations are designed so that the 

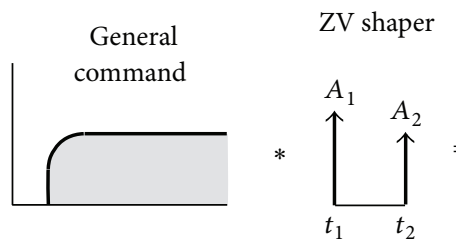

(a) ZV input shaping process

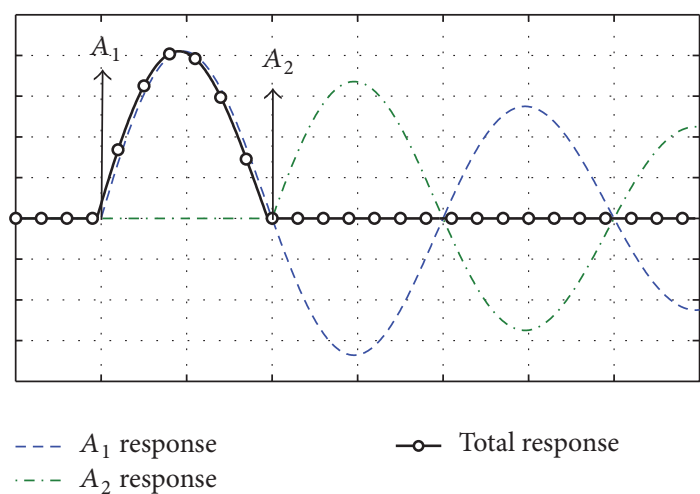

(b) Impulse response to the $\mathrm{ZV}$ input shaper

FIGURE 3: Zero-vibration (ZV) input shaping.

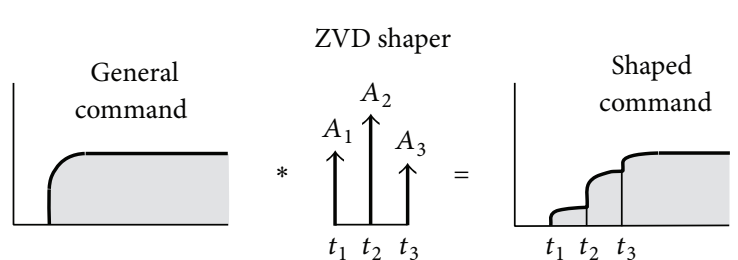

(a) ZV input shaping process

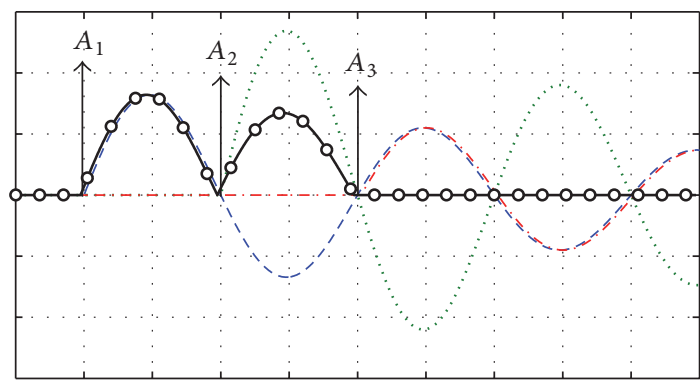
--- $A_{1}$ response
-.- $A_{3}$ response
- - Total response

(b) Impulse response to the ZVD input shaper

FIGURE 4: Zero-vibration-derivative (ZVD) input shaping.

residual vibration is zero [31]. The impulse amplitudes and impulse times of the ZV input shaper are

$$
\mathrm{ZV}=\left[\begin{array}{c}
A_{i} \\
t_{i}
\end{array}\right]=\left[\begin{array}{cc}
\frac{1}{1+K} & \frac{K}{1+K} \\
0 & \frac{\tau_{d}}{2}
\end{array}\right]
$$

where $A_{i}$ and $t_{i}$ are the $i$ th impulse amplitude and time, respectively, and where $\tau_{d}$ is the damped period of oscillation, and

$$
K=e^{-\zeta \pi / \sqrt{1-\zeta^{2}}}
$$

where $\zeta$ is the damping ratio.

The robust ZVD input shaping process is the convolution of three impulses with a general input command, Figure 4. The ZVD impulses and their time locations are designed so that the amplitude and the derivative of residual oscillations caused by the shaped input are zero [31]. This results in an input shaper in the form

$$
\begin{aligned}
\mathrm{ZVD} & =\left[\begin{array}{c}
A_{i} \\
t_{i}
\end{array}\right] \\
& =\left[\begin{array}{ccc}
\frac{1}{1+2 K+K^{2}} & \frac{2 K}{1+2 K+K^{2}} & \frac{K^{2}}{1+2 K+K^{2}} \\
0 & \frac{\tau_{d}}{2} & \tau_{d}
\end{array}\right] .
\end{aligned}
$$

Generally, the use of ZVD shaper is preferable for its robustness and low sensitivity; however, the shaper time length is double that of the $\mathrm{ZV}$ shaper.

\section{Numerical Simulations}

To demonstrate the performance of the proposed FM shaper, several simulations are presented in this section. The model is driven using a step acceleration command with an amplitude of $1 \mathrm{~m} / \mathrm{s}^{2}$. The maximum velocity of the jib is set to $0.6 \mathrm{~m} / \mathrm{s}$. The hoist acceleration and velocity are set to $1 \mathrm{~m} / \mathrm{s}^{2}$ and 


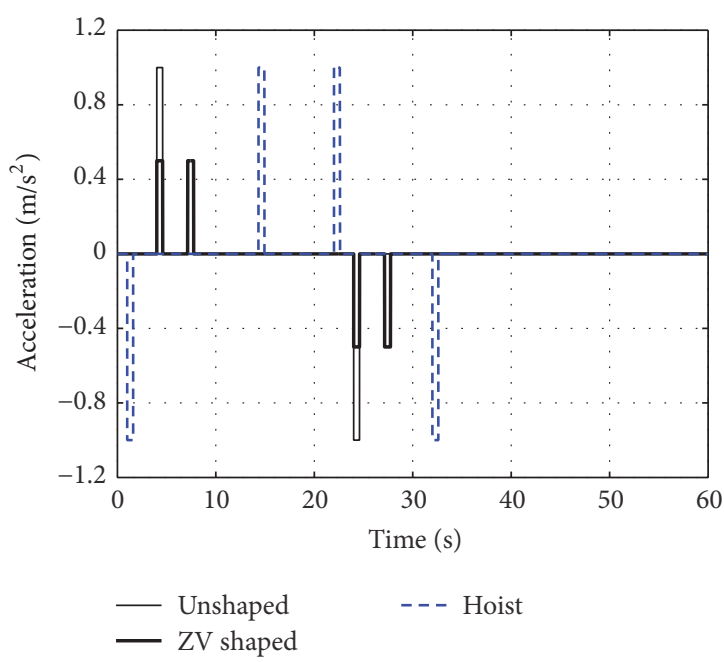

(a) Acceleration commands

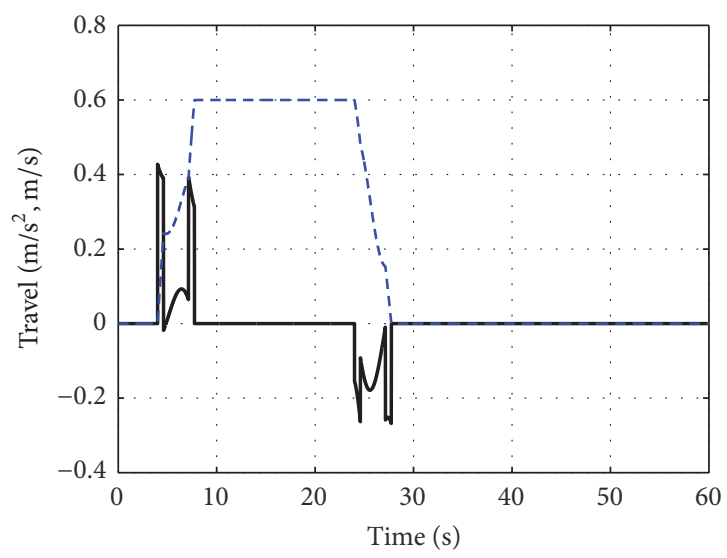

ZV + FM acceleration
$---\mathrm{ZV}+$ FM velocity

(c) FM acceleration and velocity

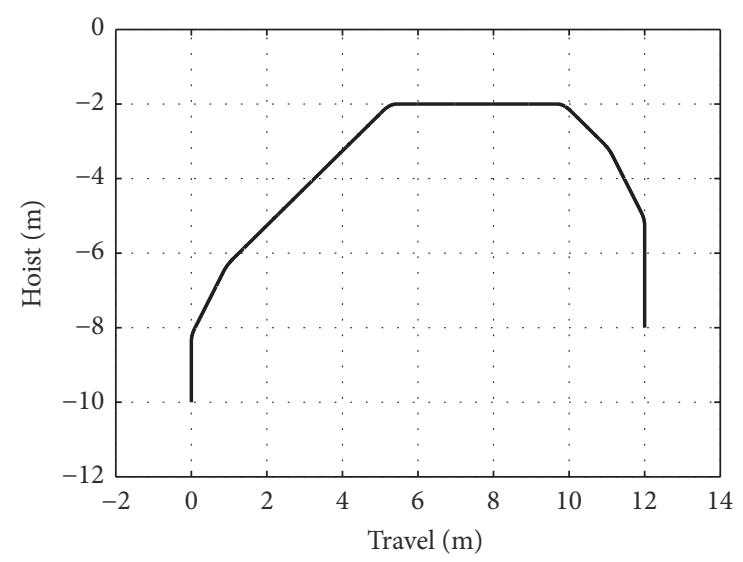

(b) Payload trajectory

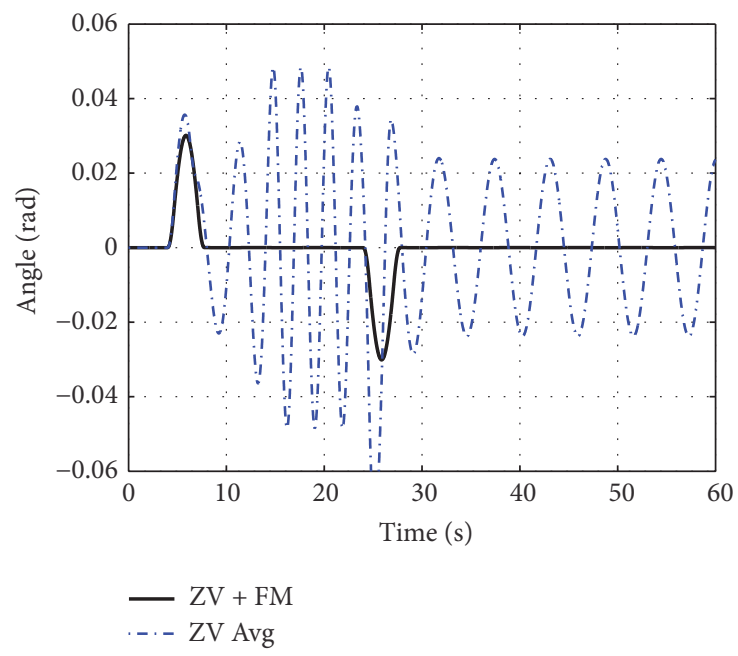

(d) Payload oscillation angle

FIGURE 5: Simultaneous travel and hoisting maneuver using FM shaped command with primary ZV input shaper.

$0.6 \mathrm{~m} / \mathrm{s}$, respectively. The minimum length of the hoisting cable is set to $2 \mathrm{~m}$ while the maximum length is set to $10 \mathrm{~m}$. The design frequency of the FM shaper is set to $1 \mathrm{rad} / \mathrm{s}$ which, approximately, corresponds to the maximum length of the hoisting cable. The design model of the FM shaper is assumed undamped. Using (8) and (10), the resulting ZV and ZVD shapers are

$$
\begin{aligned}
\mathrm{ZV} & =\left[\begin{array}{ll}
\frac{1}{2} & \frac{1}{2} \\
0 & \pi
\end{array}\right] \\
\mathrm{ZVD} & =\left[\begin{array}{lll}
\frac{1}{4} & \frac{1}{2} & \frac{1}{4} \\
0 & \pi & 2 \pi
\end{array}\right] .
\end{aligned}
$$

Figure 5(a) shows the jib and hoist acceleration commands. The figure also shows the jib acceleration command after it is passed through the primary ZV shaper. The resulting payload trajectory is in Figure 5(b). The initial cable length is set to $10 \mathrm{~m}$. The payload is hoisted up $8 \mathrm{~m}$ to a level $2 \mathrm{~m}$ under the jib and then lowered $6 \mathrm{~m}$ down to a level $8 \mathrm{~m}$ under the jib. Simultaneously, the jib performs a $12 \mathrm{~m}$ travel maneuver. The FM model-based feedback causes the frequency of the model to remain fixed at its design value of $1 \mathrm{rad} / \mathrm{s}$, which makes it possible to use a single-mode ZV shaper. The combined ZV acceleration command with the FM feedback and the corresponding jib velocity are shown in Figure 5(c). A comparison of the payload response oscillation angle using the ZV-based FM shaper and a ZV shaper designed for the average oscillation frequency of the maneuver [17] is presented in Figure 5(d). Figure 5(d) shows that the payload travel and residual oscillations are virtually eliminated using the FM shaper. The time cost of the ZV primary shaping is only one-half the design period of approximately $3 \mathrm{s.}$ 


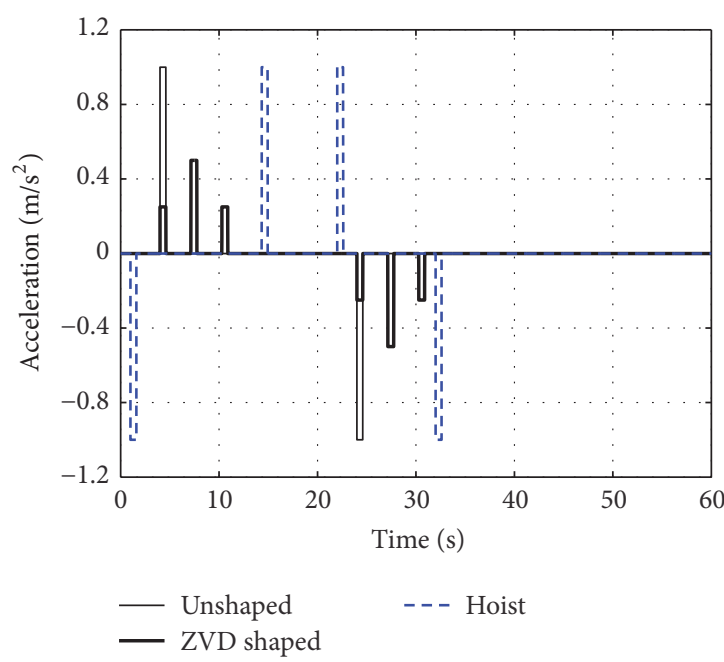

(a) Acceleration commands

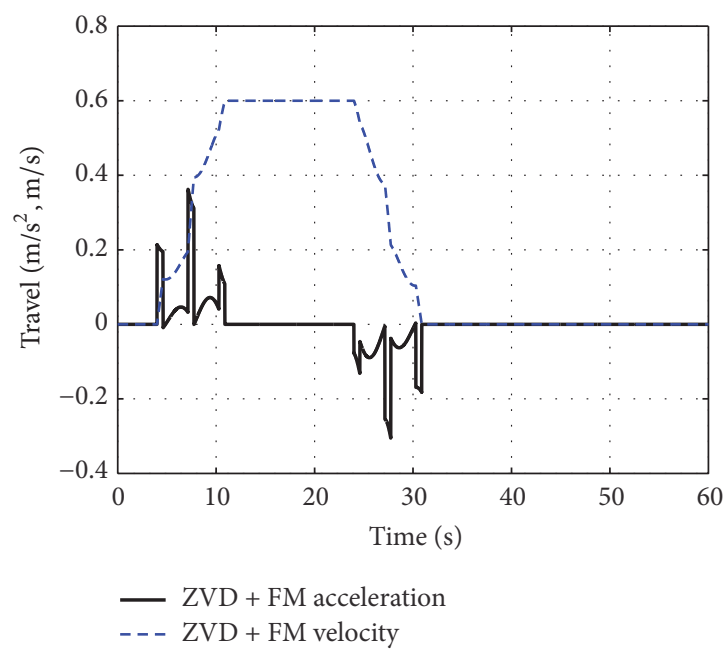

(c) FM acceleration and velocity

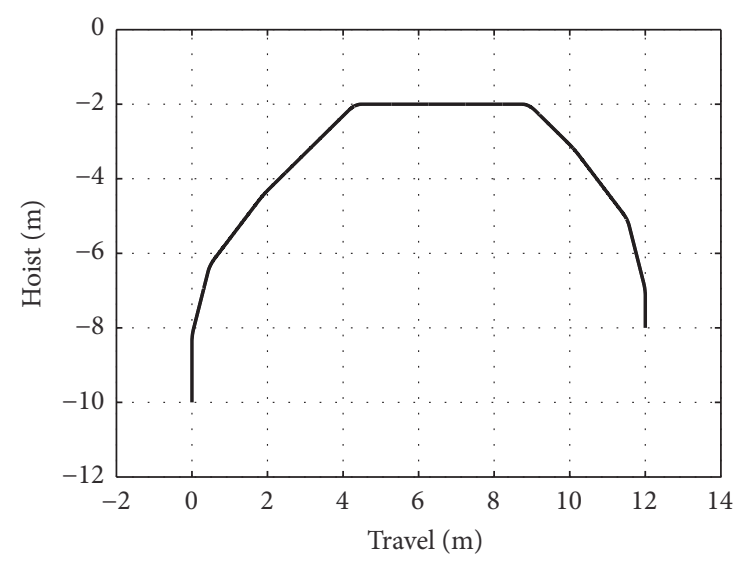

(b) Payload trajectory

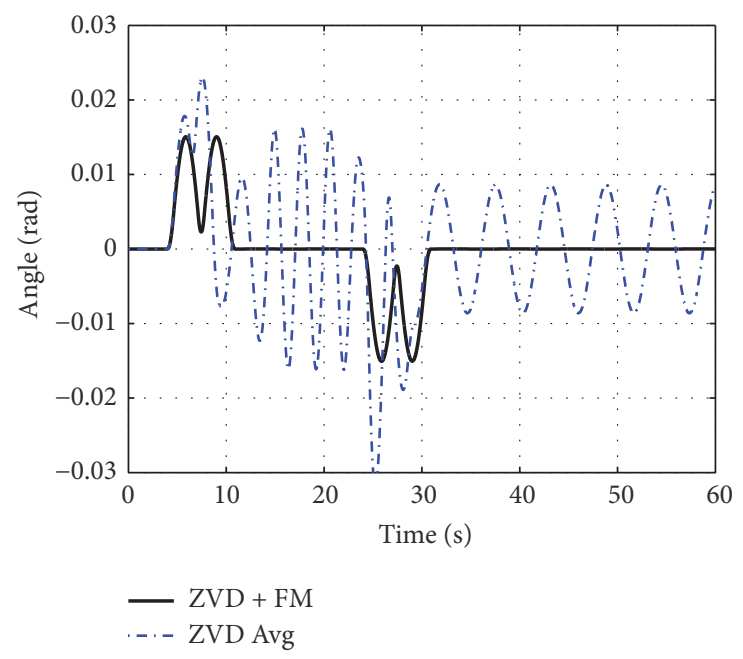

(d) Payload oscillation angle

FIGURE 6: Simultaneous travel and hoisting maneuver using FM shaped command with primary ZVD input shaper.

The same maneuver is repeated using the ZVD primary input shaper in (12). Figure 6 shows the same satisfactory performance as in the case of the ZV primary input shaper, Figure 5. Results of both cases in Figures 5 and 6 demonstrate the fact that the FM shaping scheme allows large and fast hoisting during travel maneuvers and the fact that FM shaping can accommodate different primary input shapers. The time penalty of using the ZVD primary shaping is only one design period, which is approximately $6 \mathrm{~s}$.

Results also demonstrate one major advantage of using FM shaping, which is the fact that one single-mode input shaper can be used to eliminate oscillations in systems with continuously time varying frequency. This advantage eliminates the need to use higher order input shapers that involve large number of impulses and include large time penalties in the order of several periods of oscillation in the shaping process $[31,32]$.
Numerous maneuver profiles were tested using both ZVand ZVD-based FM shapers. During those tests, a residual drift in the jib position was observed when a change in the hoisting speed occurs during the command shaping phase. In Figures 7(a) and 7(b), the shaped deceleration starts at $24 \mathrm{~s}$. The lowering of the payload starts at $25 \mathrm{~s}$ causing hoist and no hoist situations to coexist during the primary ZV and ZVD shaping stages. This change in the hoisting speed caused the FM command to not integrate to zero, which resulted in small residual jib velocity, Figures 7 (c) and 7(d). This residual jib velocity caused the jib position to continue to drift slowly after the conclusion of the travel command. Nevertheless, this drift did not have any effect on the performance of the FM shaper in terms of eliminating travel and residual oscillation, Figures 7(e) and 7(f). Applying the jib brakes during this drift is observed to result in small residual oscillations. 


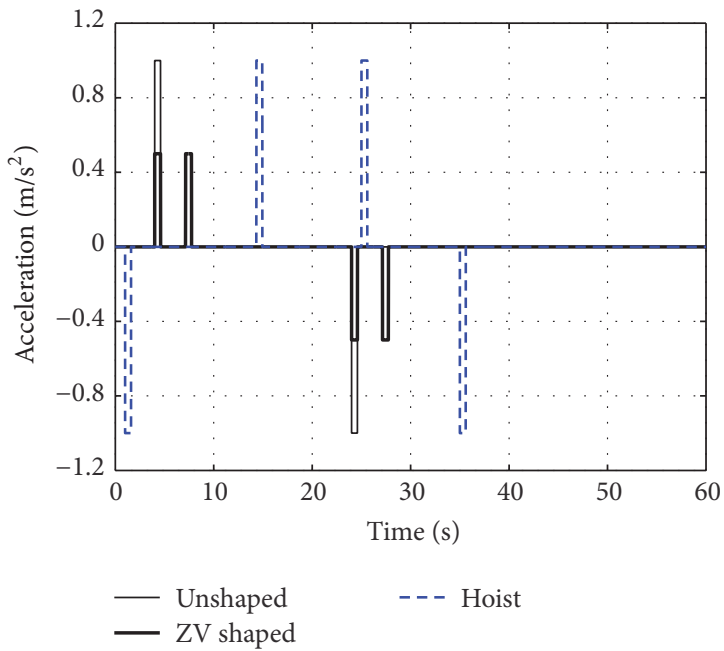

(a) ZV acceleration commands

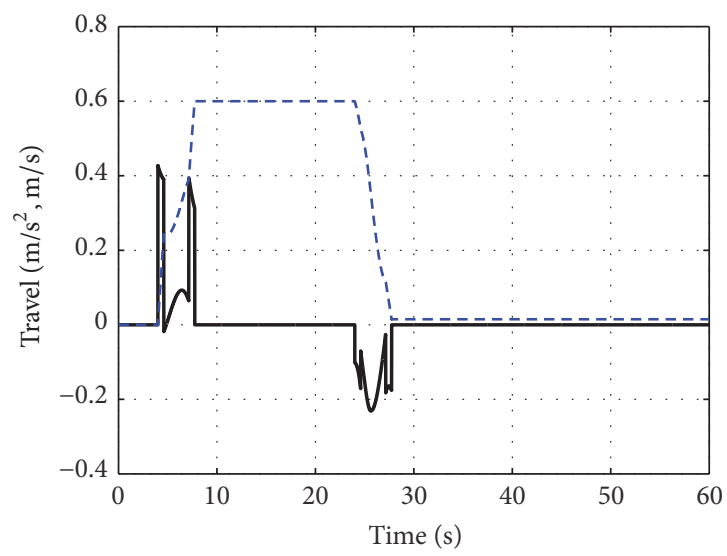

- $\mathrm{ZV}+\mathrm{FM}$ acceleration

- - - ZV + FM velocity

(c) ZV + FM acceleration and velocity

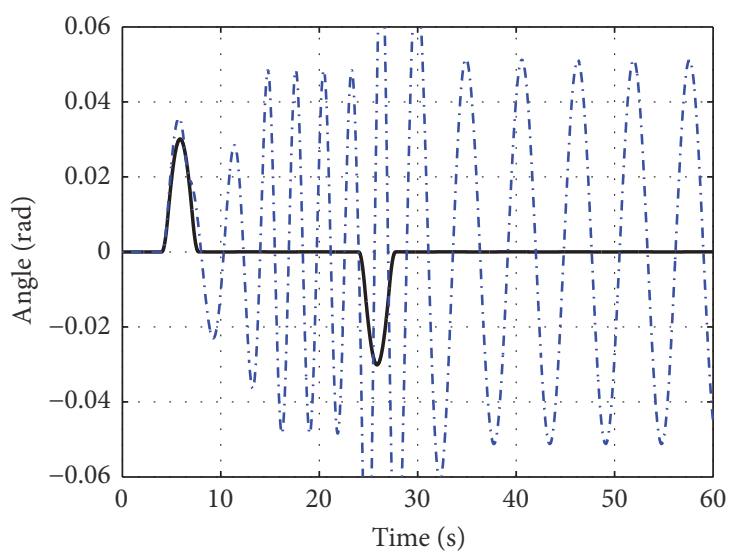

$-\mathrm{ZV}+\mathrm{FM}$

...- ZV Avg

(e) ZV + FM payload oscillations

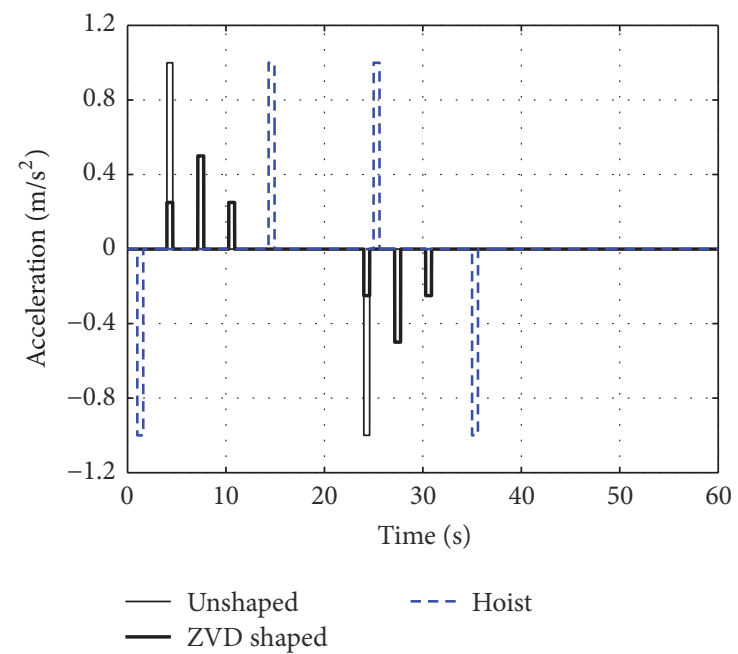

(b) ZVD acceleration commands

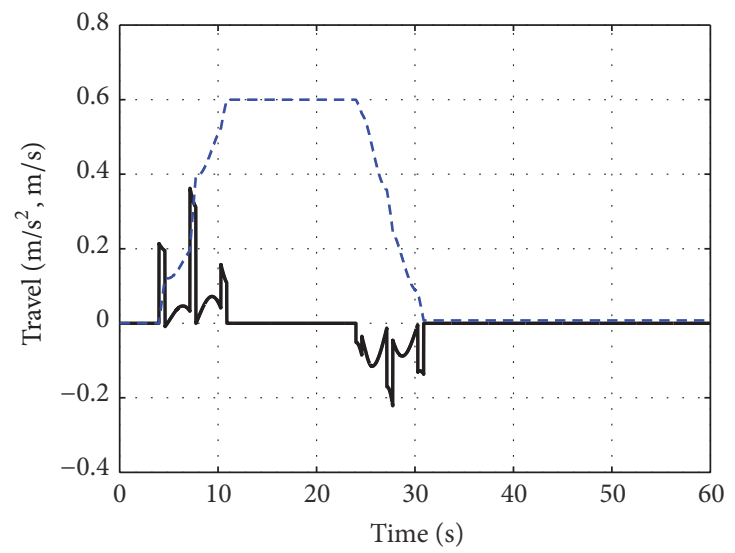

- ZVD + FM acceleration

- - - ZVD + FM velocity

(d) ZVD + FM acceleration and velocity

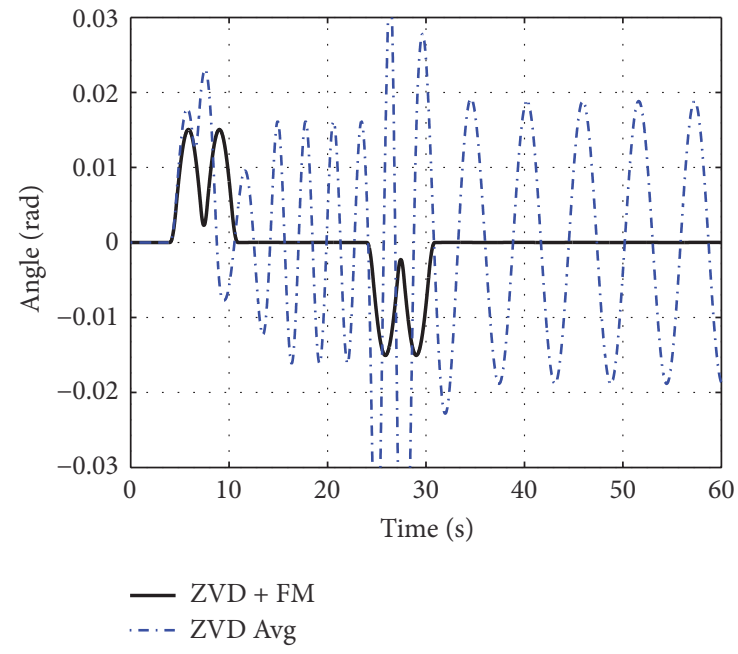

(f) ZVD + FM payload oscillations

FIGURE 7: Simultaneous travel and hoisting maneuver using FM shaped command. 


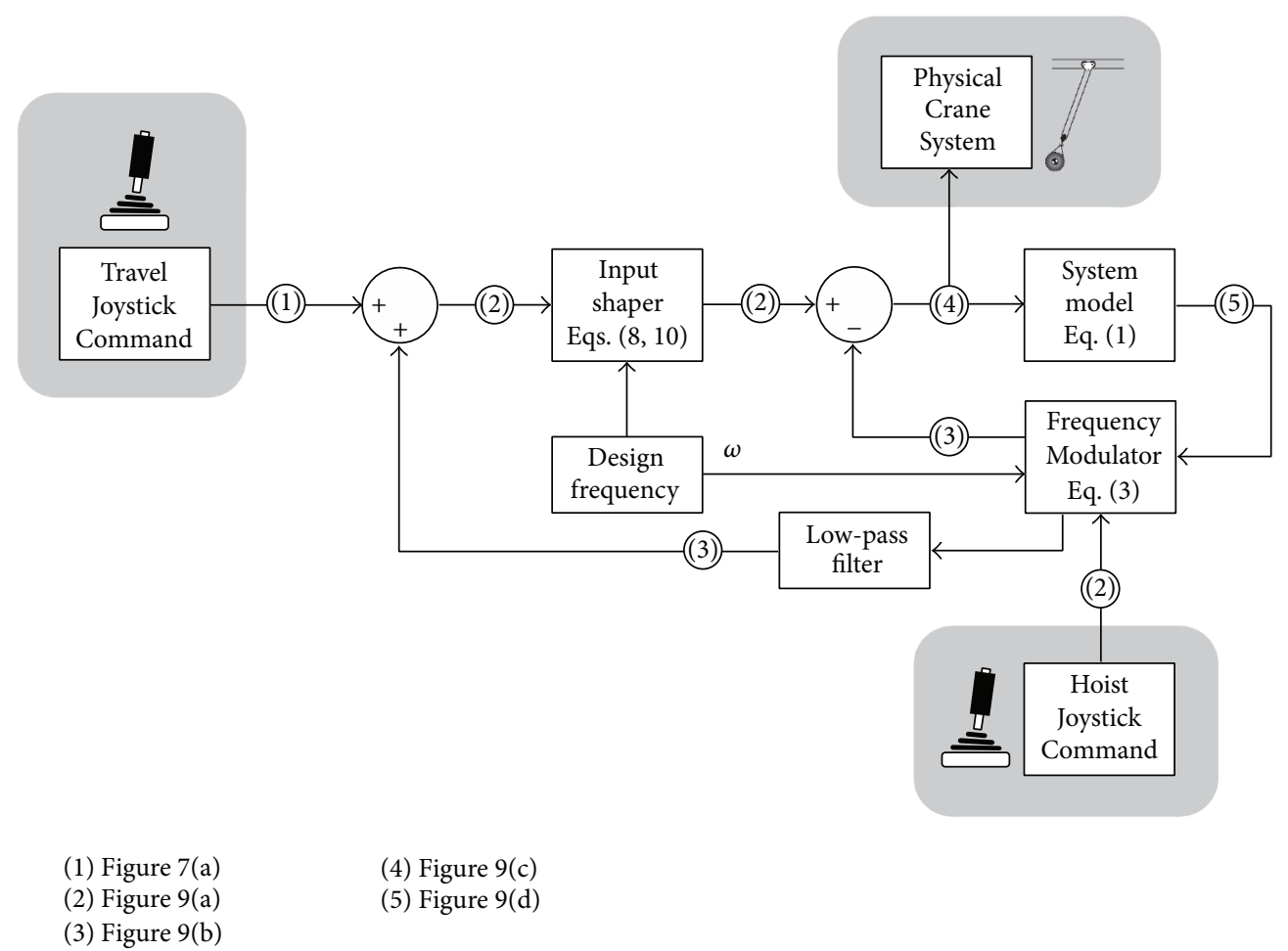

FIGURE 8: Schematic block diagram of the modified frequency modulation input shaping.

To eliminate the effect of the FM feedback on the total motion of the jib, a modified FM shaping scheme is devised, Figure 8. The FM command is added to the initial travel command prior to the shaping stage. The positive FM feedback before the input shaper balances the negative FM feedback to the model which eliminates the drift problem. The positive FM feedback correction does not affect the FM shaping process since the primary input shaper deals with this signal as a part of the general input command. The positive FM feedback is routed through a low-pass filter to minimize jerks in the preshaper command. The low-pass filter used is a first-order Chebyshev II with a stopband edge to 60 times the design frequency of the primary shaper $(10 \mathrm{~Hz}$ in these simulations) and a stopband attenuation of $40 \mathrm{~dB}$.

The test case in Figure 7 is repeated using the modified FM shaping scheme, Figure 9. Residual jib motion is completely eliminated, Figure 7(c). A transient payload oscillation is observed at the positive FM feedback correction; however, the amplitude of this transient oscillation is less than $10 \%$ $\left(0.5^{\circ}\right)$ of the uncontrolled response and rapidly dies out. The time penalty incurred due to this correction is one shaping cycle, which, in the case of ZV primary shaping, is approximately one-half period of the FM shaper design period, Figure $7(\mathrm{~d})$.

To further verify the effectiveness of the modified FM shaping scheme, an arbitrary hoist command is performed during a travel maneuver. The resulting payload trajectory is shown in Figures 10(a) and 10(b). Continuous large and arbitrarily changing hoist commands are entered during the shaped acceleration and deceleration stages of the maneuvers, Figures $10(\mathrm{c})$ and $10(\mathrm{~d})$. There are small transient oscillations in the correction phases in the order of $3^{\circ}$ with the $\mathrm{ZV}$ primary shaper and $1.5^{\circ}$ in the case of primary ZVD shaper, Figures 10(e) and 10(f). In both cases, the transient oscillations die out in one primary shaper cycle. In all practicality, due to the small amplitude of the correction transient oscillation, those extra shaping cycles do not add up to the settling time of the maneuver.

\section{Sensitivity Analysis}

The main problem with traditional input shaping techniques is their sensitivity to the design frequency or set of frequencies of the shaper. Technologies currently used for measuring the actual hoisting cable length and the hoisting speeds are accurate. However, the arbitrary location of the center of mass of the crane payload introduces uncertainty in determining the effective length of the hoisting cable. To investigate the impact of this uncertainty on the performance robustness of the FM input shaping scheme, an offset in the cable length measurement was used in determining the feedback gain (7).

Several maneuvers are tested for different values of measurement offset. Figure 11(a) shows five arbitrary payload trajectories. The length of the hoisting cable is varied between $1 \mathrm{~m}$ and $10 \mathrm{~m}$. Results showed that residual oscillations using the FM shaper are not affected by the maneuver trajectory. All five trajectories yielded identical residual oscillations for the same measurement offset, Figure 11(b). 


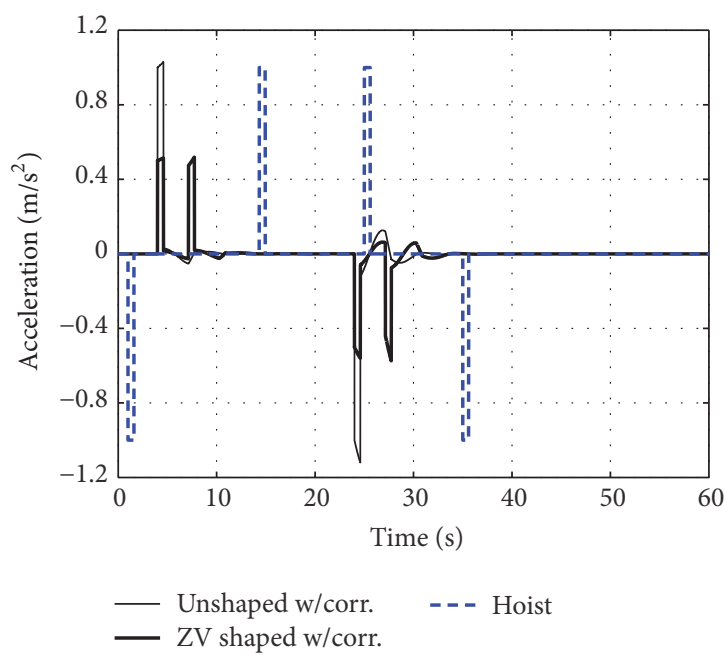

(a) Corrected acceleration commands

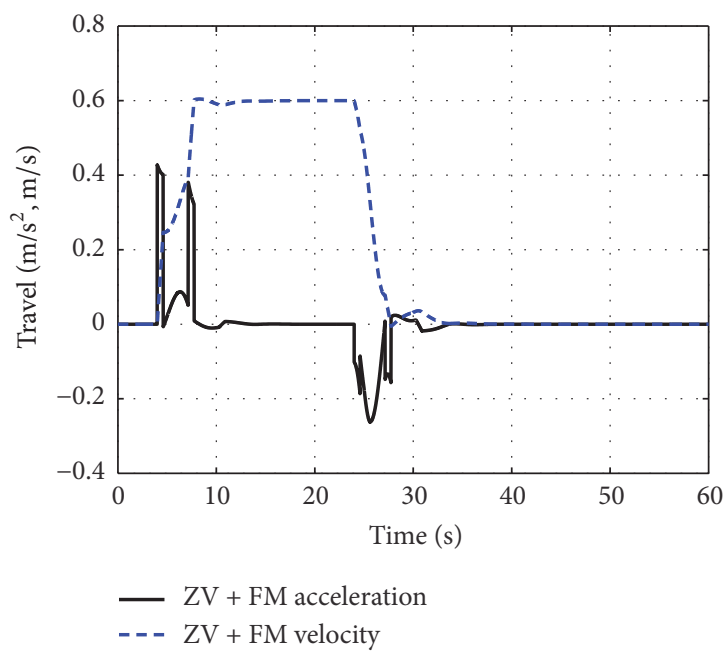

(c) FM acceleration and velocity

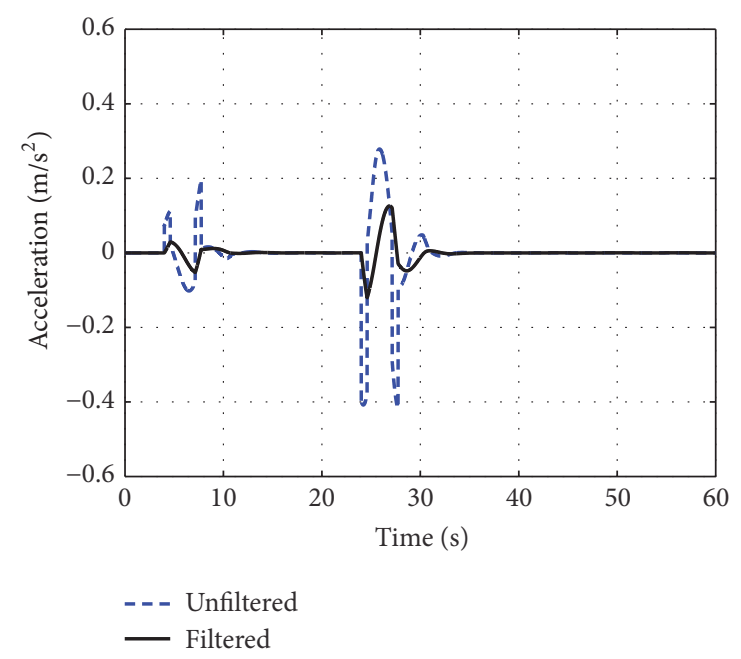

(b) Positive FM feedback

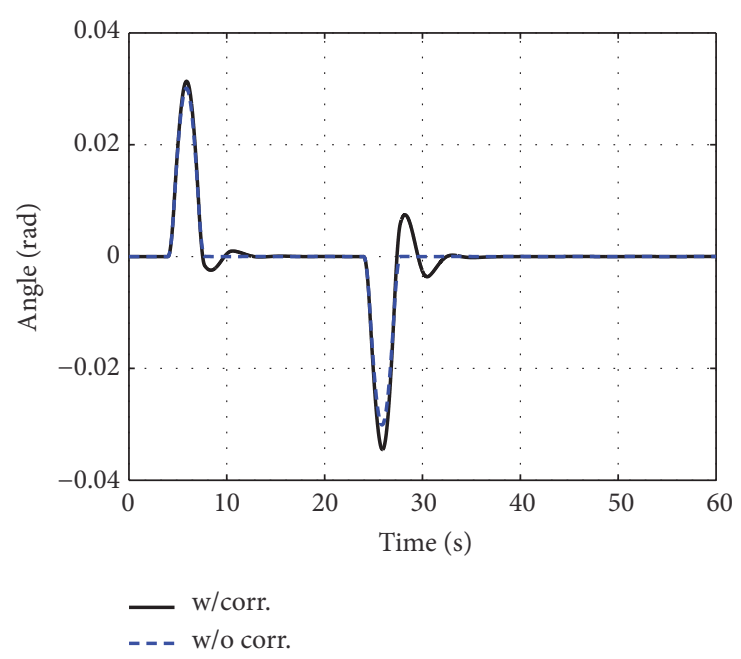

(d) Payload oscillation angle

FIGURE 9: Simultaneous travel and hoisting maneuver using modified FM shaping with primary ZV input shaper.

Sensitivity curves of the FM shaper with ZV and ZVD primary input shapers are shown in Figure 12. For 5\% residual oscillations tolerance, Figure 12(b), the FM shaper with ZV primary input shaper demonstrates a $\pm 10 \%$ offset tolerance relative to the hoisting cable length associated with the design frequency $\omega$ of the FM shaper. The offset tolerance range of the FM shaper with ZVD primary input shaper is significantly large and ranging from $-60 \%$ to $25 \%$ of the design cable length of the FM shaper. This demonstrates a significant improvement over the traditional ZVD shaper which has an offset tolerance range of approximately $\pm 17 \%$ [31].

\section{Concluding Remarks}

In this paper a frequency modulation input shaping scheme is proposed to facilitate simultaneous travel and hoisting maneuvers in overhead cranes. This scheme allows using single frequency input shapers that tend to be faster than multiple-frequency input shapers and include fewer number of impulses. A major advantage of the proposed scheme is the flexibility in continuously changing the hoisting cable length without restrictions. In some cases where the hoisting speed changes during the input shaping process, a small drift in jib's position is observed at the end of maneuvers. To eliminate this drift, an additional modified FM input shaping scheme is proposed. The FM command is added to the unshaped travel command to remove any residual velocity offset usually occurring when a change in the hoisting speed takes place during the command shaping stage. The modified FM shaping scheme eliminates restrictions on where and when you start the hoisting process making the proposed scheme even more effective. Another major advantage of this scheme is the significantly reduced sensitivity of the FM + ZVD shaper when compared to the traditional ZVD shaper. 


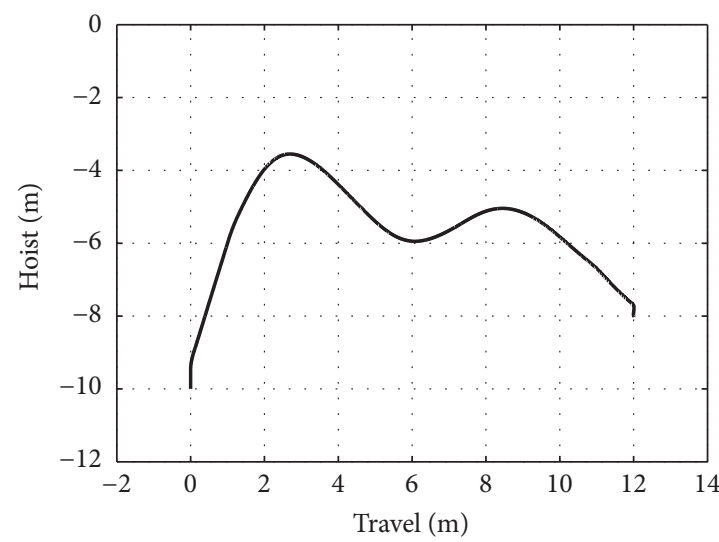

(a) ZV payload trajectory

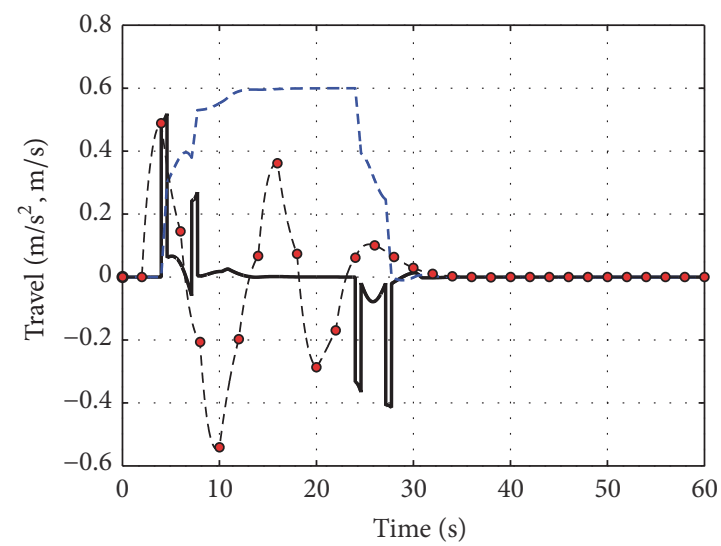

- ZV + FM acceleration

- - ZV + FM velocity

- - Hoist acceleration

(c) ZV + FM and hoist accelerations

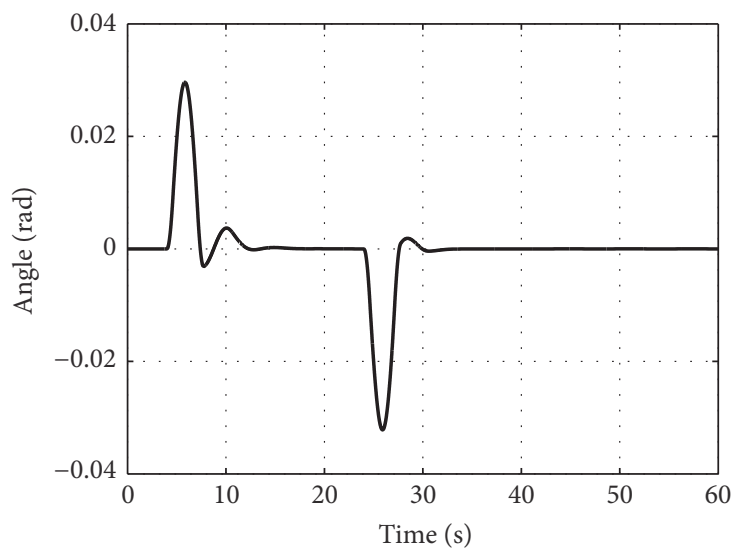

(e) ZV + FM payload oscillations

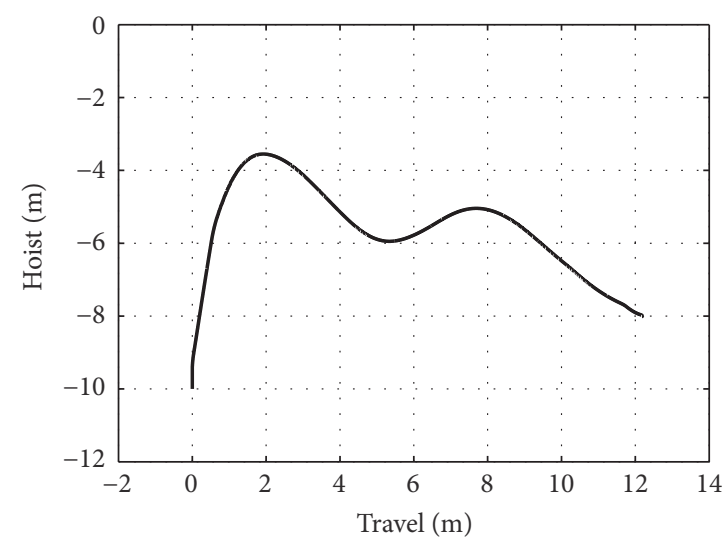

(b) ZVD payload trajectory

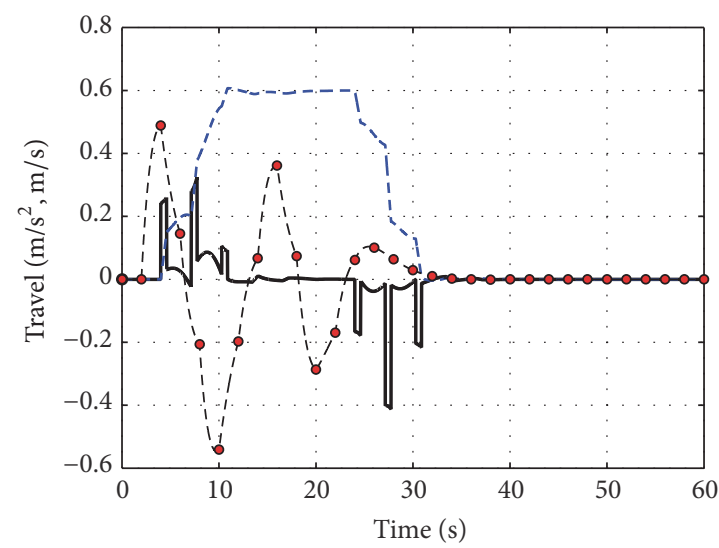

- ZVD + FM acceleration

- - - ZVD + FM velocity

- - Hoist acceleration

(d) ZVD + FM and hoist accelerations

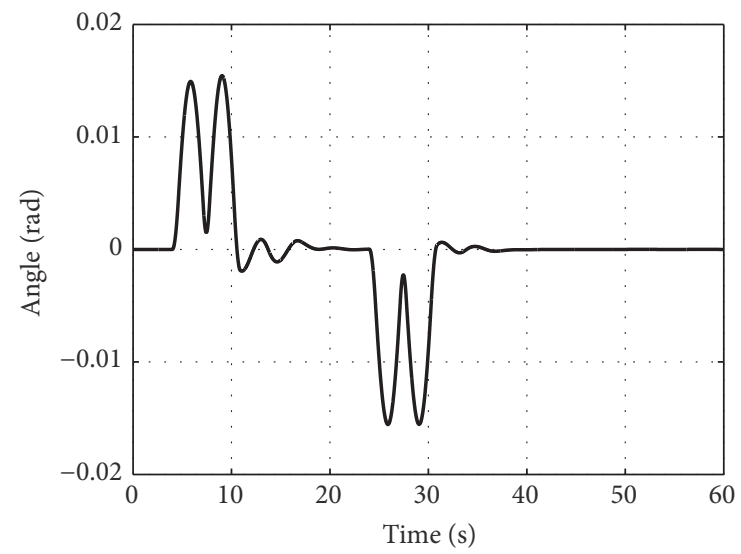

(f) ZVD + FM payload oscillations

FIGURE 10: Simultaneous travel and hoisting maneuver using FM shaped command. 


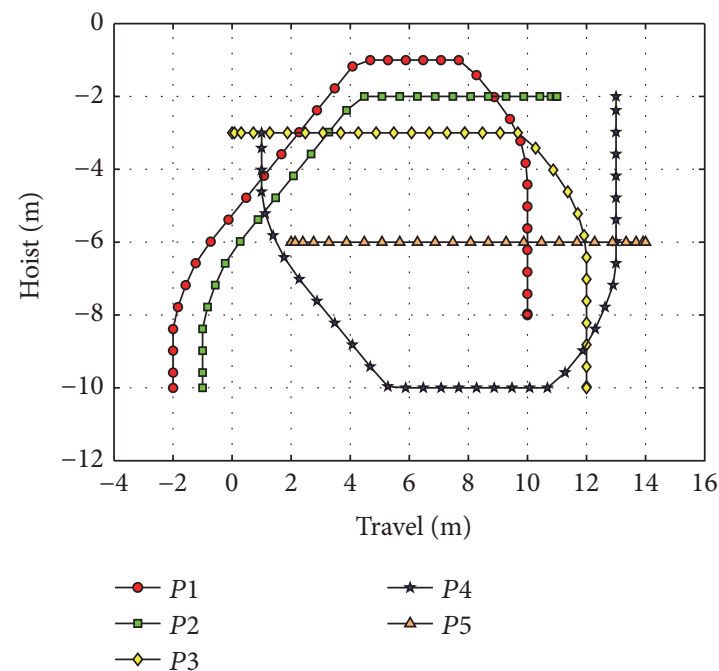

(a) Payload trajectory

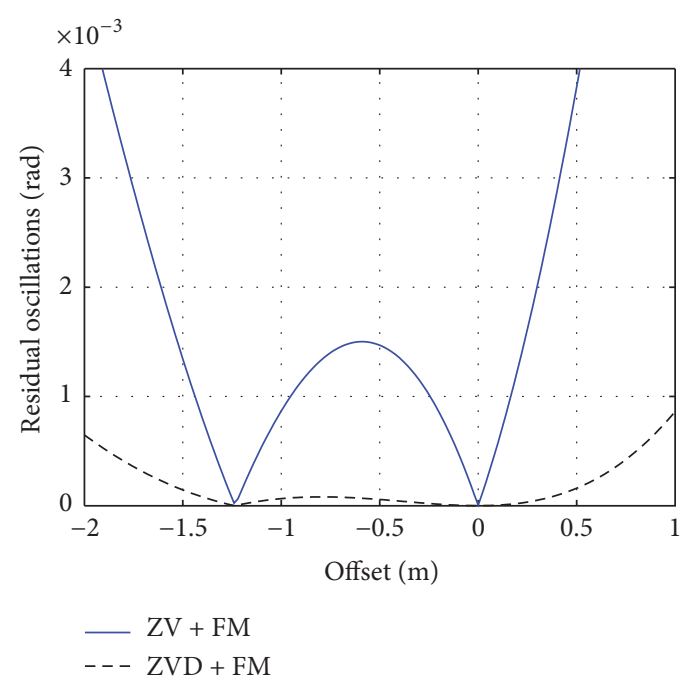

(b) Residual oscillations

FIGURE 11: Simultaneous travel and hoisting maneuver using FM shaped command.

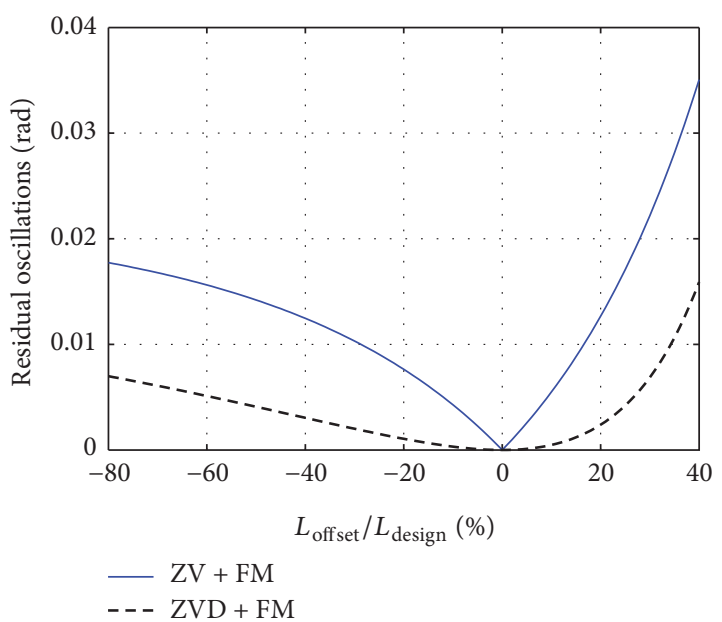

(a) Residual oscillations

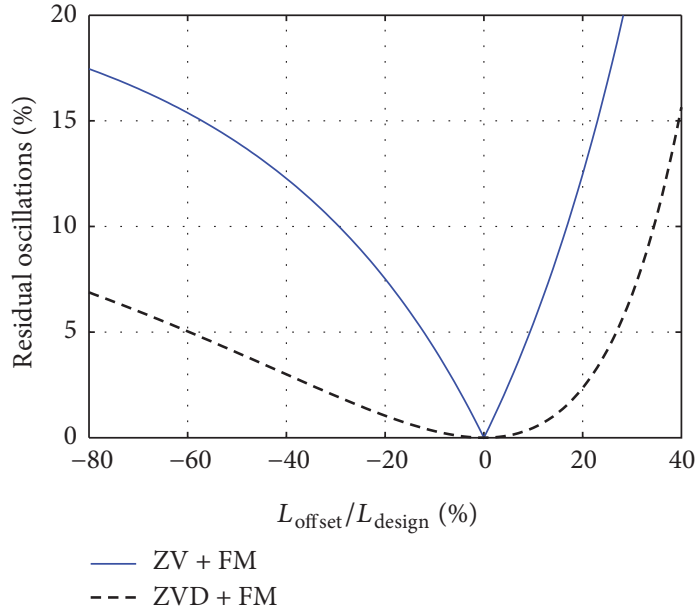

(b) Percentage residual oscillations

FIGURE 12: Simultaneous travel and hoisting maneuver using FM shaped command.

As a result the system is much more tolerant to cable length measurement errors resulting from different payload shapes and sizes.

\section{Conflicts of Interest}

The authors declare that there are no conflicts of interest regarding the publication of this paper.

\section{References}

[1] E. M. Abdel-Rahman, A. H. Nayfeh, and Z. N. Masoud, "Dynamics and control of cranes: a review," Journal of Vibration and Control, vol. 9, no. 7, pp. 863-908, 2003.
[2] W. Singhose, "Command shaping for flexible systems: a review of the first 50 years," International Journal of Precision Engineering and Manufacturing, vol. 10, no. 4, pp. 153-168, 2009.

[3] Z. N. Masoud, A. H. Nayfeh, and D. T. Mook, "Cargo pendulation reduction of ship-mounted cranes," Nonlinear Dynamics, vol. 35, no. 3, pp. 299-311, 2004.

[4] Z. N. Masoud, A. H. Nayfeh, and N. A. Nayfeh, "Sway reduction on quay-side container cranes using delayed feedback controller: simulations and experiments," Journal of Vibration and Control, vol. 11, no. 8, pp. 1103-1122, 2005.

[5] S.-K. Cho and H.-H. Lee, "A fuzzy-logic antiswing controller for three-dimensional overhead cranes," ISA Transactions, vol. 41, no. 2, pp. 235-243, 2002. 
[6] G. P. Starr, "Swing-free transport of suspended objects with a path-controlled robot manipulator," Journal of Dynamic Systems, Measurement, and Control, vol. 107, no. 1, pp. 97-100, 1985.

[7] D. R. Strip, "Swing-Free Transport of Suspended Suspended Objects: A General Treatment," IEEE Transactions on Robotics and Automation, vol. 5, no. 2, pp. 234-236, 1989.

[8] M. A. Ahmad, R. M. T. R. Ismail, M. S. Ramli, N. M. A. Ghani, and N. Hambali, "Investigations of feed-forward techniques for anti-sway control of 3-D gantry crane system," in Proceedings of 2009 IEEE Symposium on Industrial Electronics and Applications, ISIEA 2009, pp. 265-270, mys, October 2009.

[9] N. Singer, W. Singhose, and W. Seering, "Comparison of filtering methods for reducing residual vibration," European Journal of Control, vol. 5, no. 2-4, pp. 208-218, 1999.

[10] T. Singh, "Jerk limited input shapers," in American Control Conference, Proceedings of the 2004, IEEE, pp. 4825-4830, 2004.

[11] W. Singhose, R. Eloundou, and J. Lawrence, "Command generation for flexible systems by input shaping and command smoothing," Journal of Guidance, Control, and Dynamics, vol. 33, no. 6, pp. 1697-1707, 2010.

[12] K. Erkorkmaz and Y. Altintas, "High speed CNC system design. Part I. Jerk limited trajectory generation and quintic spline interpolation," International Journal of Machine Tools and Manufacture, vol. 41, no. 9, pp. 1323-1345, 2001.

[13] K. A. Alhazza and Z. N. Masoud, "A novel wave-form command-shaping control with application on overhead cranes," in Proceedings of ASME 2010 Dynamic Systems and Control Conference, DSCC2010, pp. 331-336, usa, September 2010.

[14] K. Alhazza and Z. Masoud, "A novel wave-form commandshaper for overhead cranes," Journal of Engineering Research, vol. 1, no. 3, pp. 181-209, 2013.

[15] K. Alhazza, A. Al-Shehaima, and Z. Masoud, "A continuous modulated wave-form command shaping for damped overhead cranes," in Proceedings of the ASME 2011 International Design Engineering Technical Conferences \& Computers and Information in Engineering Conference, pp. 361-366, Washington, DC, USA, 2011.

[16] K. A. Alhazza, "Experimental validation on a continuous modulated wave-form command shaping applied on damped systems," in Proceedings of 31st IMAC, A Conference on Structural Dynamics, 2013, pp. 445-451, usa, February 2013.

[17] W. Singhose, L. Porter, M. Kenison, and E. Kriikku, "Effects of hoisting on the input shaping control of gantry cranes," Control Engineering Practice, vol. 8, no. 10, pp. 1159-1165, 2000.

[18] Z. N. Masoud and M. F. Daqaq, "A graphical approach to inputshaping control design for container cranes with hoist," IEEE Transactions on Control Systems Technology, vol. 14, no. 6, pp. 1070-1077, 2006.

[19] K. A. Alhazza, "Experimental and numerical validation on a continuous modulated wave-form command shaping control considering the effect of hoisting," in Proceedings of the ASME 2013 International Design Engineering Technical Conferences \& Computers and Information in Engineering Conference, Portland, Oregon, USA, 2013, pp. V07AT10A057.

[20] K. A. Alhazza, A. M. Hasan, K. A. Alghanim, and Z. N. Masoud, "An iterative learning control technique for point-topoint maneuvers applied on an overhead crane," Shock and Vibration, vol. 2014, Article ID 261509, 11 pages, 2014.
[21] K. A. Alghanim, K. A. Alhazza, and Z. N. Masoud, "Discretetime command profile for simultaneous travel and hoist maneuvers of overhead cranes," Journal of Sound and Vibration, vol. 345, pp. 47-57, 2015.

[22] Y. Zhou, X. Zhang, Z. Yu, D. Schott, and G. Lodewijks, "An improved zero vibration method and parameter sensitivity analysis for the swing control of bridge-type grab ship unloader," Proceedings of the Institution of Mechanical Engineers, Part C: Journal of Mechanical Engineering Science, vol. 230, no. 14, pp. 2463-2472, 2016.

[23] A. M. Abdullahi, Z. Mohamed, M. Z. Abidin et al., "Outputbased command shaping technique for an effective payload sway control of a 3D crane with hoisting," Transactions of the Institute of Measurement and Control, 2016, pp. 0142331216640871.

[24] M. W. Spong, "Underactuated mechanical systems," in Control Problems in Robotics and Automation, pp. 135-150, Springer, 1998.

[25] C.-C. Cheng and C.-Y. Chen, "Controller design for an overhead crane system with uncertainty," Control Engineering Practice, vol. 4, no. 5, pp. 645-653, 1996.

[26] B. Fang and A. Kelkar, "On feedback linearization of underactuated nonlinear spacecraft dynamics," in Proceedings of the 40th IEEE Conference on Decision and Control, IEEE, 2001.

[27] X. Zhang, B. Gao, and H. Chen, "Nonlinear controller for a gantry crane based on partial feedback linearization," in Proceedings of 5th International Conference on Control and Automation, IEEE (ICCA '05), 2005.

[28] H. Park, D. Chwa, and K.-S. Hong, "A feedback linearization control of container cranes: Varying rope length," International Journal of Control, Automation and Systems, vol. 5, no. 4, pp. 379-387, 2007.

[29] H. C. Cho and K. S. Lee, "Adaptive control and stability analysis of nonlinear crane systems with perturbation," Journal of Mechanical Science and Technology, vol. 22, no. 6, pp. 10911098, 2008.

[30] T. A. le, S.-G. Lee, and S.-C. Moon, "Partial feedback linearization and sliding mode techniques for 2D crane control," Transactions of the Institute of Measurement and Control, vol. 36, no. 1, pp. 78-87, 2014.

[31] J. Vaughan, A. Yano, and W. Singhose, "Comparison of robust input shapers," Journal of Sound and Vibration, vol. 315, no. 4-5, pp. 797-815, 2008.

[32] R. Kumar and T. Singh, "Design of input shapers using modal cost for multi-mode systems," Automatica. A Journal of IFAC, the International Federation of Automatic Control, vol. 46, no. 3, pp. 598-604, 2010. 


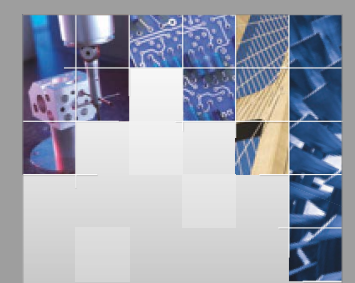

\section{Enfincering}
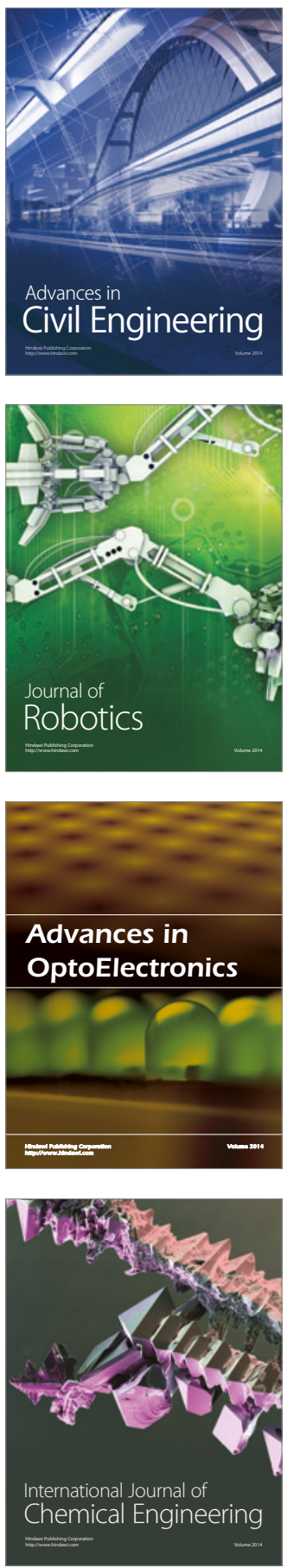

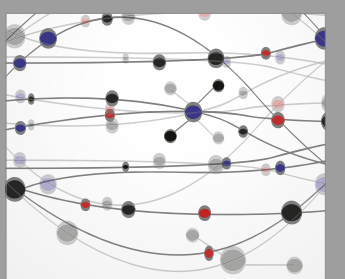

The Scientific World Journal

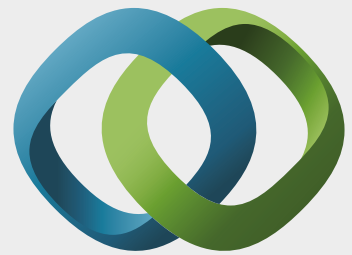

\section{Hindawi}

Submit your manuscripts at

https://www.hindawi.com
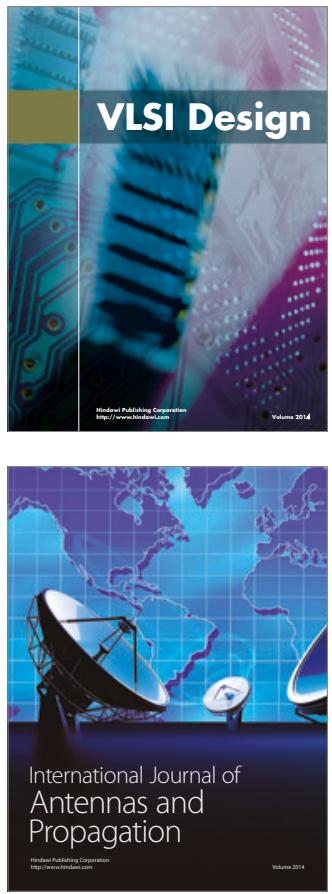

\section{Rotating}

Machinery
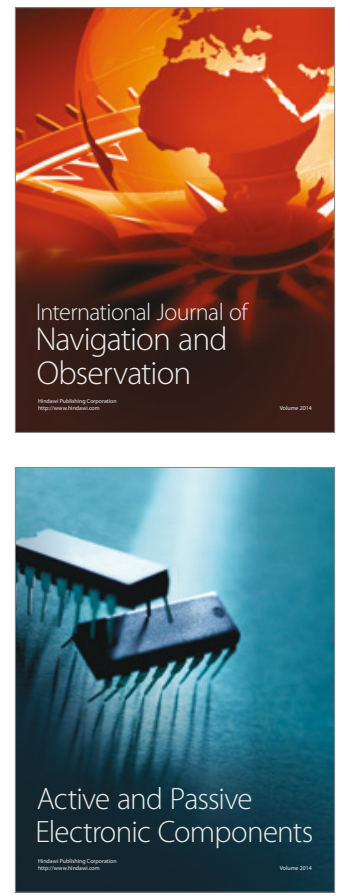
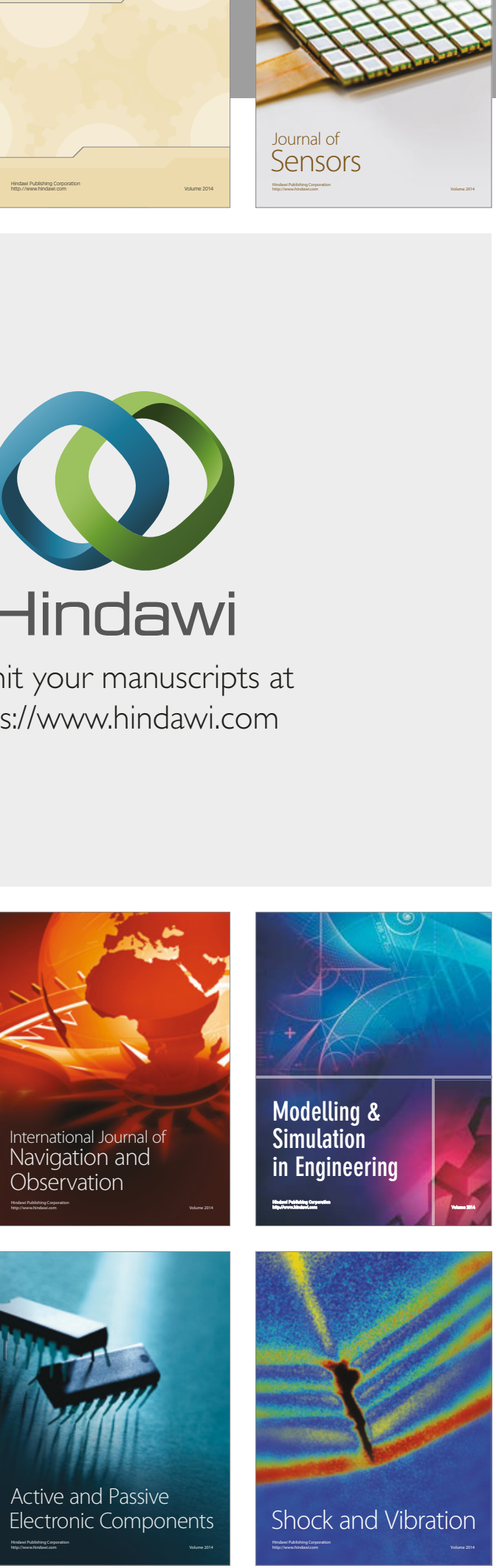
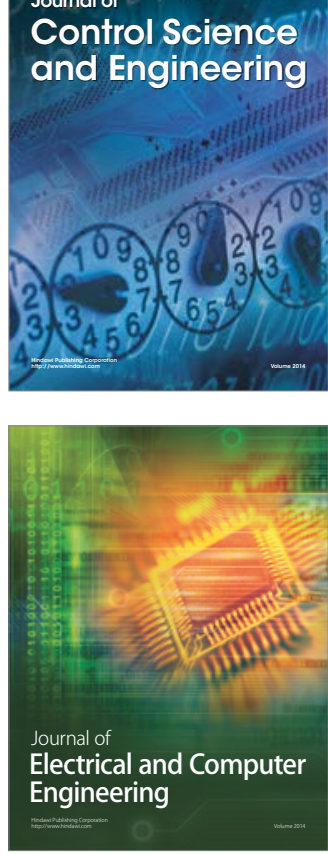

Distributed

Journal of

Control Science

and Engineering
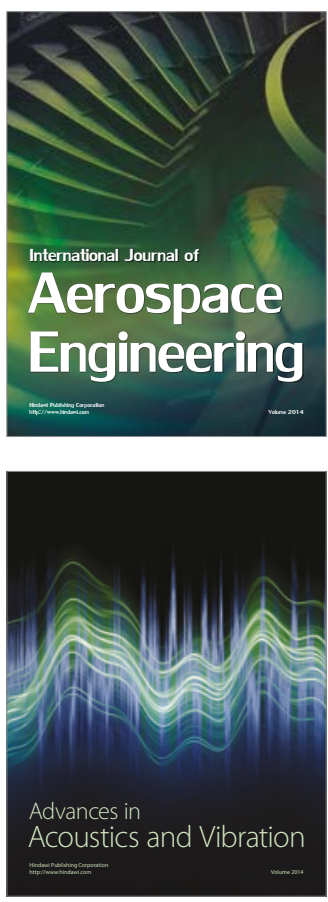

Sensor Networks 